\title{
Diversity and Aboveground Carbon Sequestration Potential of On-Farm Agroforestry Trees/shrubs Species in Rajaf County-South Sudan
}

\author{
Joseph Mayindo Mayele ${ }^{1 *}$ \\ ${ }^{1}$ College of Natural Resources and Environmental Studies, \\ University of Juba, P.o.Box +82 , Juba, \\ South Sudan \\ John Bosco Lamoris Okullo ${ }^{2}$ \\ ${ }^{2}$ School of Forestry, Environmental and Geographical \\ Sciences, Makerere University, P.o.Box 7062, Kampala, \\ Uganda
}

\begin{abstract}
Agroforestry (AF) trees have the potentials to sequester carbon in most farming systems especially in the face of the current climate change. The objective of this study was to estimate the diversity, abundance, and aboveground carbon sequestration potential of onfarm tree species in four different sites, Tokiman, Gumbo, Kolye West, and Kolye East with various agroforestry practices in Rajaf County, South Sudan. Three hundred and fifty-five AF trees were inventoried by non-destructive methods for diameter at breast height (DBH), height (H) and Crown width (CR) variables. Aboveground biomass analyzed using allometric models was converted to carbon stocks. The Shannon-Weiner indices for the AF sites indicated that $C$. limon, $M$. indica and $P$. guajava were more diverse in Tokiman and Gumbo sites. $T$. grandis was more diverse in Kolye East and Kolye West, with $S$. siamea only diverse in Kolye West. The diversity analyses also showed low values of (H') for Gumbo and Tokiman as compared to those calculated for Kolye West and Kolye East. In addition, high levels of aboveground carbon sequestration were observed in Gumbo and Kolye East sites where tree species were relatively abundant. Kolye West, on the other hand, had very few but highly dominant AF tree species while Tokiman Islands with the most planted tree species had a rare agro-ecology that contributed less or nearly zero to aboveground carbon sequestration.
\end{abstract}

Keywords:- Diversity index, carbon sequestration, agroforestry, above-ground biomass, Rajaf County, South Sudan.

\section{INTRODUCTION}

Climate change has become a real aspect of global environmental concern. It is nowadays a real and major threat to many rural communities around the globe. There is a growing interest in the role of different land use systems in stabilizing carbon dioxide in the atmosphere [1]; [2]. Therefore, forest land cover is considered an important resource for mitigating of the harmful effects of the carbon

\author{
Susan Balaba Tumwebaze ${ }^{2}$ \\ ${ }^{2}$ School of Forestry, Environmental and Geographical \\ Sciences, Makerere University, P.o.Box 7062, Kampala, \\ Uganda \\ Augustine LokuleBongo ${ }^{1}$ \\ ${ }^{1}$ College of Natural Resources and Environmental Studies, \\ University of Juba, P.o.Box +82 , Juba, \\ South Sudan
}

dioxide emissions due to human activities through photosynthesis [3]; [4].

Worldwide, terrestrial ecosystems sequester about $22.2 \%$ flux of fossil fuels annually [5]. In the context of climate change, forest ecosystems and its material content account for $48 \%$ of the total storage capacity of carbon by worldwide terrestrial ecosystems [6]; [7]. As such forests contain about $80 \%$ of global terrestrial above ground biomass and are important carbon sinks [8]; [9]. Forest therefore, are the best reservoir of carbon because of their capacity to stock potentially large amounts of carbon from the atmosphere through its material content.

Tree biomass are the major constituents of all the forest ecosystems that absorbs large amounts of atmospheric carbon dioxides by photosynthesis, and can return seemingly the same amounts to the atmosphere by autotrophic or heterotrophic respiration [10]. Assessment of biomass, which includes above-ground and below-ground living and non-living mass allows for forest valuation for the purpose of estimating carbon and available resources for energy.

However, most of the previous research work on biomass estimation found it difficult to collect field data of below ground biomass [11]; [12]. This is because belowground biomass is not immediately affected by common deforestation and degradation processes, unlike aboveground biomass. As a useful measure in assessing changes in forest structure and an essential aspect of carbon cycles, above-ground estimates have always been a source of uncertainty of carbon balance from the tropical regions of Africa. This is partly due to variations in landscape and forest types coupled to scarcity of reliable estimates of live aboveground biomass [13]; [14].Therefore, for proper extrapolation of biomass stocks to ecosystems, improved estimates of these AGB would provide essential data [13].

Although agroforestry systems have high potential to generate ecosystem services, they accrue little and inexplicably lower attention than conventional forest ecosystems [15]. There is limited data on contribution of 
agroforestry systems to carbon sequestration in subSaharan Africa, for instance in South Sudan and in Rajaf county in particular. Therefore, such inadequate data are deemed to undermine smallholders' potential on agroforestry practices for sequestering carbon [16].

Agroforestry traditionally includes trees under different systems, including agrisilvicultural, silvopastoral and agrisilvopastoral systems [17]. These systems components include perennials such as trees, shrubs, vines, crops and other herbaceous species, and animals. Practices of this traditional agroforestry include woodlots, boundary planting, homegardens, scattered trees in farmlands, planting as hedgerows, alley cropping, taungya systems, and shifting cultivation among others.

Trees in agroforestry farms contributes to soil protection, water regulation, enhancement of microclimatic conditions, reduces impacts on natural forests and other environmental benefits in addition to its major climate regulation function (carbon sequestration) [18]. When trees are integrated on farms, improvements in land productivity and resilience of households has been shown through products diversification for human sustainability [19].

The use of allometric equations for estimation of tree biomass is the most appropriate option since the study involved non-destructive methods of measuring AGB. In this case, tree variables such as diameter at breast height $(\mathrm{DBH})$, height $(\mathrm{H})$ and crown widths $(\mathrm{CR})$ are measured in addition to their estimated wood densities. The variables were then fitted in the existing allometric equations [20]; [21]. According to reference [22], allometric models usually yield estimates which are less biased if total tree height is available. Inspite of this, tree heights has always been ignored in carbon-estimation exercises because it is difficult to take height measurements accurately especially in agroforestry land uses. This is because most trees do not have accurate architectural patterns to determine their heights.

There have been serious controversies in the global change societies as to whether or not tree heights must be included as one of the predictors of AGB [23]. Studies done by [24] found out that including height, wood density or crown width in biomass equation changed the biomass estimates by a trivial amount, less than $1.2 \mathrm{mg}$ or $1.3 \%$ of total biomass, from those obtained by using diameter alone. This finding is in conformity with most research studies [25]; [26]. In this context, use of DBH alone for estimating AGB appears cost-effective and robust for most purposes in comparison to the complexities and potential errors in measuring height. Therefore, because of its easiness in measurement and high accuracy, [27] considered diameter as the most widely preferred predictor variable especially in on-farms agroforestry trees that explains approximately over $95 \%$ of variability observed in AGB.

\section{MATERIALS AND METHODS}

\section{$>$ StudyArea}

The study was conducted in the four payams, Gumbo, Kolye West, Kolye East and Tokiman Islands of Rajaf County, Jubek State-South Sudan. Rajaf County is located along the eastern and western banks of River Nile, southeast direction from Juba city at latitude range between $4^{\circ}$ and $6^{\circ}$ North and longitude of $27^{\circ}$ and $32^{\circ}$ East respectively. It covers a total area of $3,204 \mathrm{Km}^{2}$ and hosts a total population of about 15,604 people [28]. The area is covered by open woodland and grasslands in the rich moist tropical and highland ranges.

The climate in the area is characterized by rainfall intensity and its humidity that usually exceeds $80 \%$ during the rainy season, and drops to below $50 \%$ in the dry season [29]; [30]. Temperatures range between $30^{\circ} \mathrm{C}-33^{\circ} \mathrm{C}$ all year round especially in the dry season, and drops to at least an average of $18^{\circ} \mathrm{C}$ in the wet season [31].

The study sites located near the shores of Nile river are also characterized with nutrient-rich soils that are suitable for agriculture as well as growth of agroforestry species and natural forests stands.

\section{$>$ Sampling Design}

The study was based on on-farm inventory with circular sample plots laid along a line transect. At least three (3) subsequent line transects of approximately $50 \mathrm{~m}$ apart were placed systematically across each agroforestry farm of $\geq 0.5$ ha. A minimum of four (4) circular sample plots of equal radius $(12.6 \mathrm{~m})$ and $30 \mathrm{~m}$ apart were then established along each transect according to [32]. The plot design is more appropriate for this type of study because marking of the plot boundaries is not necessary [33]. The agroforestry farms and trees within the circular sample plots were randomly selected and were subjected to nondestructive method of biomass measurement and direct observation. This was used to obtain tree variables of diameter at breast height $(\mathrm{DBH})$, heights $(\mathrm{H})$ and crown width (CR).

\section{$>$ Diameter at breast height $(D B H)$, height $(H)$ and crown width (CR) measurements}

A non-destructive method was used for measuring the diameter at breast height $(\mathrm{DBH})$, height $(\mathrm{H})$ and crown width (CR) which are essential variables for assessing species diversity, abundance, and carbon sequestration potentials of AF trees in the study sites. In each sample plot, measurements were carried out solely on trees/shrubs with $\mathrm{DBH} \geq 5 \mathrm{~cm}$ and height of $\geq 1.3 \mathrm{~m}$ as described in [34]. The reason for using the $\mathrm{DBH} \geq 5 \mathrm{~cm}$ was because AF trees in the study areas were quite young whose stems $\geq 5 \mathrm{~cm}$ would be measured quite easily and that most developed allometric equations use $\mathrm{DBH}$ from $\geq 5 \mathrm{~cm}$. The $\mathrm{DBH}$ of trees/shrubs were measured using a caliper for trees with small diameters, or diameter tape for trees with larger diameters; and height was measured using a Suunto Clinometer [35]. Crown widths were also measured at ground level in two directions of North-South and East- 
West using measuring tape. All tree species encountered were identified according to species name, and species family using species identification guide and their frequency of occurrence recorded as described in previous studies [32].

\section{* Data Analysis}

\section{Woody Species Diversity}

Shannon diversity indices (Shannon-Wiener index) in Minitab Ver. 14 was used to analyze species diversity

The formula for Shannon-weiner diversity index is as below:

Shannon-Weiner Index $(\mathrm{H})=-\sum_{\mathrm{i}=1}^{\mathrm{s}}[\mathrm{Pi} * \ln (\mathrm{Pi})]$

Where $\mathrm{P}=$ proportion $(\mathrm{n} / \mathrm{N})$ of individuals of one particular species found (n) divided by the total number of individuals found $(\mathrm{N}) ; \mathrm{Ln}=$ natural $\log , \sum=$ sum of the calculations; and $s=$ number of species [36]

Descriptive statistics was used for species abundance analysis. Frequency of total population size of trees measured was determined; and total number comprised of each species is used to calculate its relative abundance in percentage (\%). On the other hand, abundance of trees from each AF practice is obtained by the total frequency calculated of diverse tree species.

\section{Carbon sequestration}

The variables of aboveground biomass, DBH and $\mathrm{H}$, were subjected to existing allometric equations of [37]; [38]; [39] \& [40] with [20] used as a baseline model whose accuracy was compared by their outputs they provided for AGB estimation [41]. Models for data analyses were selected based on the independent variables in each equation and the land cover from which the models were derived (Table 1). This was with the assumption that the independent variables can possibly lead to errors if used for estimation of biomass and carbon stocks across the agroforestry sites. Therefore, although errors were expected, inventory collected data was fitted into different biomass estimating models to compare among these models (Table 1) and the data was then subjected to logistic regression analyses. Model with high $\mathrm{R}^{2}$-predicted, lower MSE and PRESS was then selected to determine aboveground biomass (AGB) and hence, to calculate the aboveground carbon (AGC) sequestered [13].

\begin{tabular}{|c|c|c|c|c|c|c|c|c|}
\hline $\begin{array}{l}\text { Model } \\
\text { No. }\end{array}$ & Equation & $\begin{array}{l}\text { Land cover } \\
\text { applicability }\end{array}$ & $\begin{array}{c}\text { No. of } \\
\text { trees } \\
(\mathrm{N})\end{array}$ & $\begin{array}{l}\text { Predicted } \\
\mathbf{R}^{2}(\%)\end{array}$ & $M S E$ & PRESS & DBH Range & $\begin{array}{l}\text { Source/ } \\
\text { Author }\end{array}$ \\
\hline 1. & $\begin{array}{c}\text { AGB }= \\
0.0673 *\left(\rho D^{2} \mathrm{H}\right)^{0.976} \\
\end{array}$ & Rain forests & 4004 & 96.0 & 0.357 & 136.24 & $10 \leq \mathrm{DBH} \leq 158 \mathrm{~cm}$ & $\begin{array}{c}\text { Chave et al., } \\
2014\end{array}$ \\
\hline 2. & $\mathrm{AGB}=0.0559 * \rho \mathrm{D}^{2} \mathrm{H}$ & Rain forests & 2410 & 98.9 & 0.361 & 189.63 & $5 \leq \mathrm{DBH} \leq 150 \mathrm{~cm}$ & $\begin{array}{l}\text { Chave et } \\
\text { al.,2005 }\end{array}$ \\
\hline 3. & $\mathrm{Y}=0.110 \rho \mathrm{D}^{2+c}$ & $\begin{array}{l}\text { AF species, } \\
\text { small size } \\
\text { farms, } \\
\text { relatively } \\
\text { small } \\
\text { vegetation } \\
\text { cover, Fruit } \\
\text { trees }\end{array}$ & $<1500$ & 77.7 & 2.92 & 468.81 & $5 \leq \mathrm{DBH} \leq 50 \mathrm{~cm}$ & $\begin{array}{c}\text { Ketterings et } \\
\text { al., } 2001\end{array}$ \\
\hline 4. & $\begin{array}{c}\mathrm{Y}=\exp \{- \\
\left.2.4090+0.9522 \operatorname{Ln}\left(\mathrm{D}^{2} \mathrm{HS}\right)\right\}\end{array}$ & Rain forests & & 80.5 & 1.37 & 206.89 & $5 \leq \mathrm{DBH} \leq 156 \mathrm{~cm}$ & $\begin{array}{l}\text { Brown et } \\
\text { al., } 1989\end{array}$ \\
\hline 5. & $\begin{array}{c}\mathrm{Y}=\exp \{- \\
2.134+2.53 *(\operatorname{LnD})\}\end{array}$ & $\begin{array}{l}\text { Tropical } \\
\text { forests/ rain } \\
\text { forests }\end{array}$ & 170 & 97.0 & 2.413 & 642.05 & $5 \leq \mathrm{DBH} \leq 148 \mathrm{~cm}$ & Ponce, 2004 \\
\hline
\end{tabular}

Table 1:- Existing Biomass Estimation Equations for AGB

Source: Buyinza et al., (2014); Chave et al., (2014). $\mathrm{Y}=\mathrm{AGB}=$ fresh weight $(\mathrm{kg}) ; \mathrm{D}=\mathrm{DBH}$, diameter at breast height $(\mathrm{cm}) ; \mathrm{H}=$ height $(\mathrm{m}) ; \rho=\mathrm{S}=\mathrm{W}=$ wood density; $\mathrm{N}=$ sample (number of trees); $\mathrm{c}=\mathrm{constant}=0.62 ; \mathrm{Ln}=$ Natural Log; and $\mathrm{R}^{2}=$ coefficient of multiple determinations. The fresh (wet) weight would be transformed into biomass and carbon stocks can then be estimated and determined from the biomass values generated. The biomass formula below is used:
Biomass=Fresh weight of sampling component $\mathrm{x}$ (Dry weight of the sub sample / Fresh weight of sub sample), according to Buyinza et al.(2014)

Total dry weight $\left(\mathrm{kgm}^{-2}\right)=$ Total fresh weight $(\mathrm{kg}) \mathrm{x}$ Subsample dry weight (g) / sample fresh weight (g) $\mathrm{x}$ sample area $\left(\mathrm{m}^{2}\right)$. 


\section{RESULTS}

Diversity of on-farm agroforestry tree/shrubs species

The Shannon diversity indices computed for Gumbo, Tokiman, Kolye East, and Kolye West agroforestry sites were 1.726, 1.699, 2.268, and 2.211 (Table 2, Table 3, Table 4 \&Table 5), respectively. The diversity indices indicated that $C$. limon, $M$. indica and $P$. guajava were relatively more diverse in Gumbo and Tokiman sites than in Kolye East and Kolye West sites (Table 2 and 3). In addition, Gumbo and Tokiman sites had lower values of Shannon (H') indices (1.726 and 1.699) respectively than those reported for Kolye East and Kolye West (2.268 and 2.211), respectively. On average, this means the latter sites had lower diversity although most planted tree species were more diverse compared to natural species. Overall the total on-farm trees sampled from the four agroforestry sites revealed a moderately average diversity index ( $\left.\mathrm{H}^{\prime}\right)$ of (1.976) with average evenness of 0.809.

\begin{tabular}{|c|c|c|c|c|}
\hline Tree spp & $\begin{array}{c}\text { No. of individuals } \\
(\mathbf{n})\end{array}$ & $\mathbf{P i}=\mathbf{s a m p l e / t o t a l ~}$ & $\mathbf{L n}(\mathbf{P i})$ & $\mathbf{P i}$ in $(\mathbf{P i})$ \\
\hline Azadiractha indica & 2 & 0.020 & -3.902 & -0.079 \\
\hline Heterophyllus Artocarpus & 2 & 0.020 & -3.902 & -0.079 \\
\hline Kigelia africana & 4 & 0.040 & -3.209 & -0.129 \\
\hline Citrus Limon (Lemon) & 24 & 0.242 & -1.417 & -0.344 \\
\hline Mangifera indica & 27 & 0.273 & -1.299 & -0.354 \\
\hline Citrus Sinensis (Oranges) & 6 & 0.061 & -2.803 & -0.169 \\
\hline Psidium Guajava & 27 & 0.273 & -1.299 & -0.354 \\
\hline Senna siamea (Cassia siamea) & 1 & 0.010 & -4.595 & -0.046 \\
\hline Tectona grandis & 6 & 0.061 & -2.803 & -0.169 \\
\hline Total & $\mathbf{9 9}$ & & & -1.726 \\
\hline
\end{tabular}

Table 2:- Diversity index of agroforestry tree species in Gumbo payam sites (N=99)

$\mathrm{S}$ (number of species) $=9$

$\mathrm{N}$ (Total number of individuals $)=99$

$\mathrm{H} \max (\operatorname{Ln} \mathrm{S})=\ln (9)=2.197$

$\sum(\operatorname{sum})$ of $[\mathrm{Pi} * \ln (\mathrm{Pi})]=-1.726$

$\mathrm{H}($ Diversity index $)=-\{(-0.079)+(-0.079)+(-0.129)+(-0.344)+(-0.354)+(-0.169)+(-0.354)+(-0.046)+(-0.169)\}=\mathbf{1 . 7 2 6}$

Evenness $(\mathrm{H} / \mathrm{Hmax})=1.726 / 2.197=\mathbf{0 . 7 8 5}$

\begin{tabular}{|c|c|c|c|c|}
\hline Tree spp & $\begin{array}{c}\text { No. of individuals } \\
(\mathbf{n})\end{array}$ & $\mathbf{P i =} \mathbf{s a m p l e / t o t a l ~}$ & $\mathbf{L n}(\mathbf{P i})$ & $\mathbf{P i} \mathbf{i n}(\mathbf{P i})$ \\
\hline Acacia nilotica & 2 & 0.031 & -3.497 & -0.106 \\
\hline Acacia seberiana & 4 & 0.061 & -2.803 & -0.169 \\
\hline Azadiractha indica & 2 & 0.031 & -3.497 & -0.106 \\
\hline Heterophyllus Artocarpus & 3 & 0.045 & -3.091 & -0.141 \\
\hline Citrus limon (Lemon) & 11 & 0.167 & -0.788 & -0.299 \\
\hline Mangifera indica & 30 & 0.455 & -2.244 & -0.358 \\
\hline Psidium Guajava & 7 & 0.106 & -2.398 & -0.238 \\
\hline Senna siamea & 6 & 0.091 & -0.218 \\
\hline Ximenia americana & 1 & 0.015 & -0.189 & -1.063 \\
\hline Total & $\mathbf{6 6}$ & & $\mathbf{- 1 . 6 9 9}$ \\
\hline
\end{tabular}

Table 3:- Diversity index of agroforestry tree species in Tokiman Islands site $(\mathrm{N}=66)$

$\mathrm{S}$ (number of species) $=9$

$\mathrm{N}$ (Total number of individuals $)=66$

$\mathrm{H} \max (\operatorname{Ln} \mathrm{S})=\ln (9)=2.197$

$\sum(\mathrm{sum})$ of $[\mathrm{Pi} * \ln (\mathrm{Pi})]=-1.699$

$\mathrm{H}($ Diversity index $)=-\{(-0.106)+(-0.169)+(-0.106)+(-0.141)+(-0.299)+(-0.358)+(-0.238)+(-0.218)+(-0.063)\}=\mathbf{1 . 6 9 9}$

Evenness $(\mathrm{H} / \mathrm{Hmax})=1.699 / 2.197=\mathbf{0 . 7 7 3}$

T.grandis, Z.abyssinica, B.ethiopum, B.aegyptica and V.paradoxa were more diverse in Kolye East (Table 4) than Kolye West where S.siamea, T. grandis, C. limon and M. indica were found to be diverse (Table 5). 
ISSN No:-2456-2165

\begin{tabular}{|c|c|c|c|c|}
\hline Tree spp & $\begin{array}{c}\text { No. of individuals } \\
(\mathbf{n})\end{array}$ & $\mathbf{P i}=\mathbf{s a m p l e / t o t a l}$ & $\mathbf{L n}(\mathbf{P i})$ & Pi*/n(Pi) \\
\hline Acacia seberiana & 3 & 0.06 & -2.81 & -0.17 \\
\hline Balanites aegyptica & 5 & 0.10 & -2.30 & -0.23 \\
\hline Borassus ethiopum & 6 & 0.12 & -2.12 & -0.25 \\
\hline Bridelia micrantha & 2 & 0.04 & -3.22 & -0.13 \\
\hline Vitellaria paradoxa & 5 & 0.10 & -2.30 & -0.23 \\
\hline Combretum spp & 3 & 0.06 & -2.81 & -0.17 \\
\hline Dombeya quinqueseta & 1 & 0.02 & -3.91 & -0.08 \\
\hline Grawiya mollis & 1 & 0.02 & -3.91 & -0.08 \\
\hline Kigelia africana & 1 & 0.02 & -3.91 & -0.08 \\
\hline Sclerocarya birrea & 1 & 0.02 & -3.91 & -0.08 \\
\hline Tarmarindus indica & 3 & 0.06 & -2.81 & -0.17 \\
\hline Tectona grandis & 13 & 0.26 & -1.35 & -0.35 \\
\hline Ziziphus abyssinica & 6 & 0.12 & -2.12 & -0.25 \\
\hline Total & $\mathbf{5 0}$ & & & $\mathbf{- 2 . 2 6 8}$ \\
\hline
\end{tabular}

Table 4:- Diversity index of agroforestry tree species in Kolye East Payam site (N=50)

$\mathrm{S}$ (number of species) $=13$

$\mathrm{N}$ (Total number of individuals $)=50$

$\mathrm{H} \max (\operatorname{Ln} \mathrm{S})=\ln (13)=2.565$

$\sum(\mathrm{sum})$ of $[\mathrm{Pi} * \ln (\mathrm{Pi})]=-2.268$

$\mathrm{H}($ Diversity index $)=-\{(-0.17)+(-0.23)+(-0.25)+(-0.13)+(-0.23)+(-0.17)+(-0.08)+(-0.08)+(-0.08)+(-0.08)+(-0.17)+(-0.35)+(-$

$0.25)\}=\mathbf{2 . 2 6 8}$

Evenness $=(\mathrm{H} / \mathrm{Hmax})=2.268 / 2.565=\mathbf{0 . 8 8 4}$

\begin{tabular}{|c|c|c|c|c|}
\hline Tree spp & $\begin{array}{l}\text { No. of individuals } \\
(\mathrm{n})\end{array}$ & $\mathbf{P i}=$ sample $/$ total & $\mathbf{L n}(\mathbf{P i})$ & $\mathbf{P i} * \ln (\mathbf{P i})$ \\
\hline Acacia seberiana & 9 & 0.064 & -2.744 & -0.176 \\
\hline Anona senegalensis & 2 & 0.014 & -4.248 & -0.061 \\
\hline Azadiractha indica & 4 & 0.029 & -3.555 & -0.102 \\
\hline Balanites aegyptica & 2 & 0.014 & -4.248 & -0.061 \\
\hline Borassus ethiopum & 2 & 0.014 & -4.248 & -0.061 \\
\hline Heterophyllus Artocarpus & 2 & 0.014 & -4.248 & -0.061 \\
\hline Kigelia africana & 2 & 0.014 & -4.248 & -0.061 \\
\hline Citrus limon (Lemon) & 18 & 0.129 & -2.051 & -0.264 \\
\hline Mangifera indica & 16 & 0.114 & -2.169 & -0.248 \\
\hline Persea americana & 3 & 0.021 & -3.843 & -0.082 \\
\hline Piliostigma raticulata & 1 & 0.007 & -4.942 & -0.035 \\
\hline Psidium Guajava & 10 & 0.071 & -2.639 & -0.189 \\
\hline Sclerocarya birrea & 1 & 0.007 & -4.942 & -0.035 \\
\hline Senna siamea & 33 & 0.236 & -1.445 & -0.341 \\
\hline Tarmarindus indica & 4 & 0.029 & -3.555 & -0.102 \\
\hline Tectona grandis & 31 & 0.221 & -1.508 & -0.334 \\
\hline Total & 140 & & & -2.211 \\
\hline
\end{tabular}

Table 5:- Diversity index of agroforestry tree species in Kolye West site ( $\mathrm{N}=140)$

$\mathrm{S}$ (number of species) $=16$

$\mathrm{N}$ (Total number of individuals) $=140$

$\mathrm{H} \max (\operatorname{Ln} \mathrm{S})=\ln (16)=2.773$

$\sum$ (sum) of $[\mathrm{Pi} * \ln (\mathrm{Pi})]=-2.211$

$\mathrm{H}($ Diversity index $)=-\{(-0.176)+(-0.061)+(-0.102)+(-0.061)+(-0.061)+(-0.061)+(-0.061)+(-0.264)+(-0.248)+(-0.082)+(-0.035)+(-$

$0.189)+(-0.035)+(-0.341)+(-0.102)+(-0.334)\}=\mathbf{2 . 2 1 1}$

Evenness $(\mathrm{H} / \mathrm{Hmax})=2.211 / 2.773=\mathbf{0 . 7 9 7}$ 
Abundance of on-farm tree/shrubs species among the agroforestry practices

$M$. indica, P. guajava and C. limon were most abundant in homegardens. Species of T. grandis and S. siamea were relatively abundant in woodlots (Table 6). Most species of $C$. limon were scattered in most AF farmlands. B. aegyptica, Z. abyssinica, B.ethiopum and A. seberiana constituted animals fodder in parklands. Homegardens, boundary planting, and scattered trees in farms were practices with most abundance of AF tree species (Table 6).

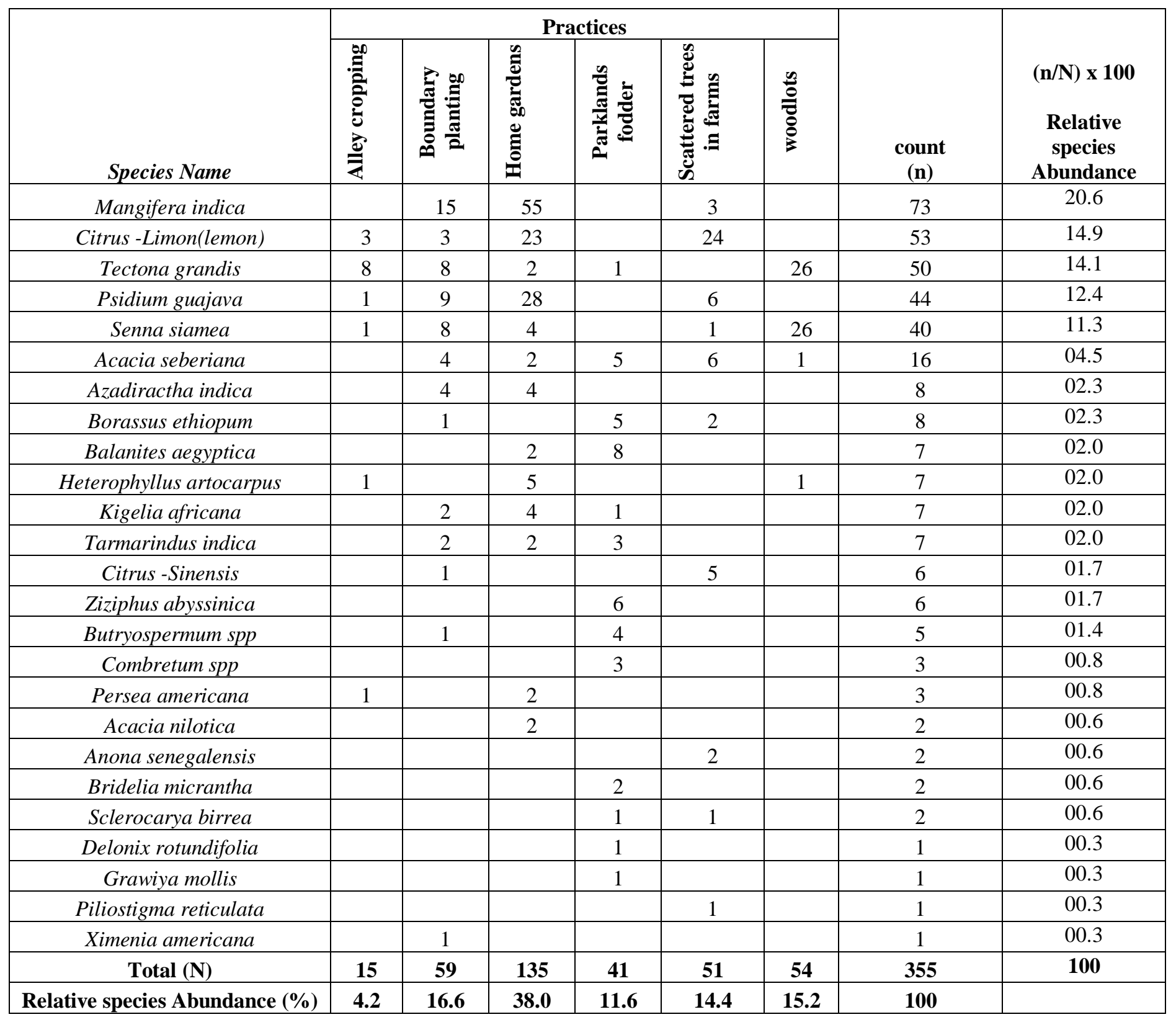

Table 6:- Species richness and abundance in various agroforestry systems/practices

- At species level: The most abundant tree species is shown by its high relative abundance (\%) of individual tree species.

- At AF practices level: The practice with most abundant species of trees is reflected by its highest total frequency (N) with diverse tree species within practice.

\section{Variables of Aboveground Biomass}

The average $\mathrm{DBH}$ and height of $\mathrm{AF}$ trees calculated was $12.68 \mathrm{~cm}$ and $6.2 \mathrm{~m}$ respectively. The minimum and maximum DBH was $5.0 \mathrm{~cm}$ and $62.9 \mathrm{~cm}$ respectively, and the minimum and maximum height $(\mathrm{H})$ was $2.3 \mathrm{~m}$ and $20.6 \mathrm{~m}$ respectively. The average crown width of $3.4 \mathrm{~m}$ was also calculated (Table 7). The meagre mean DBH and $\mathrm{H}$ indicated that trees inventoried were young, few and sparsely distributed within those AF farms. 
ISSN No:-2456-2165

\begin{tabular}{|c|c|c|c|c|c|c|c|}
\hline Variable & N & Mean \pm Std. Dev & SE Mean & Min $^{\mathbf{m}}$ & Max $^{\mathbf{m}}$ & & Median \\
\hline DBH $(\mathrm{cm})$ & 355 & $12.68 \pm 7.54$ & 0.40 & 5.00 & 62.90 & 10.30 \\
\hline Height $(\mathrm{m})$ & 355 & $06.22 \pm 2.73$ & 0.15 & 2.30 & 20.60 & 05.30 \\
\hline Average crown width $(\mathrm{m})$ & 355 & $03.38 \pm 1.05$ & 0.06 & 1.40 & 06.60 & 03.20 \\
\hline
\end{tabular}

$\mathrm{N}=$ total number of trees, Std.Dev=standard deviation, $\mathrm{SE}=$ standard error, $\mathrm{Min}^{\mathrm{m}}=$ minimum, $\mathrm{Max}^{\mathrm{m}}=$ maximum

Table 7:- Descriptive Statistics of sampled trees used in computing and comparison of models

\section{$>$ Comparison of Models}

Chave et al.(2014) model was chosen as the baseline to compare with the other models to see if the results of the computation were significant or not significant. This is because Chave et al.(2014) allometric model is mostly associated with the importance of direct heights and DBH estimations of the trees. Based on the model comparisons, the mean difference (tons/ha) for Ketterings et al.(2001) and Ponce (2004) significantly overestimated the baseline model by mean difference of -59.949 and -62.2 tons/ha ( $\mathrm{p}<0.05)$ respectively. Although, the results for Chave et al.(2005) and Brown (1989); overestimated the baseline model by respective mean difference of -65.1 and -7.3402 tons/ha, their resulting $p$-values were non-significance at $p<0.05$ (Table 8 ). The overestimation is due to the dissimilarities in the tree of height as well as trunk diameter in the allometric model which usually yields less biased estimates as compared to pantropical AGB allometries. Chave et al.(2014) and Brown (1989) models have the highest $\mathrm{R}^{2}$ (Adj.) and lowest values of MSE and PRESS (Table 9), and were therefore selected as the best models for analyzing the AGB. The values for mean AGB, $\mathrm{R}^{2}$ (Adj.), MSE and PRESS were similar for Model 1 and Model 4 (Table 9).

\begin{tabular}{|c|c|c|c|c|}
\hline Model No. & Sample size (N) & Mean difference (tons/ha) & t-value & P-value \\
\hline 1vs 2 & 355 & -65.1 & -1.77 & 0.078 \\
\hline 1vs 3 & 355 & -59.9 & -2.35 & 0.019 \\
\hline 1vs 4 & 355 & -07.3 & -0.70 & 0.484 \\
\hline 1vs 5 & 355 & -62.3 & -3.19 & 0.002 \\
\hline
\end{tabular}

Table 8:- Comparison of various models with Chave et al. (2014) as baseline model criterion

1= Chave et al.(2014); 2=Chave et al.(2005); 3= Ketterings et al.(2001); 4= Brown (1989); $5=$ Ponce (2004); $\alpha=0.05$

\begin{tabular}{|c|c|c|c|c|c|}
\hline Model Name & Model Equation & $\mathbf{N}$ & $\mathbf{R}^{\mathbf{2}} \mathbf{( \% )}$ & MSE & PRESS \\
\hline 1Chave et al.(2014) & $\mathrm{AGB}=0.0673^{*}\left(\rho \mathrm{D}^{2} \mathrm{H}\right) 0.976$ & 355 & 97.6 & 0.023 & 1.2357 \\
\hline 2Chave et al.(2005) & $\mathrm{LnY}=-1.864+2.608 \mathrm{LnD}+\mathrm{Ln} \rho$ & 355 & 53.3 & 24723 & 13311334 \\
\hline 3Ketterings et al. $(2001)$ & $\mathrm{Y}=0.110 \rho \mathrm{D} 2+\mathrm{c}$ & 355 & 62.6 & 19779 & 11262172 \\
\hline 4Brown, $(1989)$ & $\mathrm{Y}=\exp \left\{-2.4090+0.9522 \mathrm{Ln}\left(\mathrm{D}^{2} \mathrm{HS}\right)\right\}$ & 355 & 92.9 & 16 & 8683.14 \\
\hline 5Ponce, $(2004)$ & $\mathrm{Y}=\exp \{-2.134+2.53 *(\mathrm{LnD})\}$ & 355 & 69.0 & 16409 & 8568126 \\
\hline
\end{tabular}

Table 9:- Comparing regression parameters to determine biomass model to select

Where $\mathrm{Y}=\mathrm{AGB}=$ fresh weight $(\mathrm{kg}) ; \mathrm{D}=\mathrm{DBH}$ in $\mathrm{cm}$, diameter at breast height; $\mathrm{H}=$ height in $\mathrm{m} ; \rho=\mathrm{S}=\mathrm{W}=$ wood density; $\mathrm{n}=$ sample (number of trees); $\mathrm{c}=$ constant $=0.62 ; \mathrm{Ln}=$ Natural $\mathrm{Log} ; \mathrm{R}^{2}=$ Coefficient of multiple determination, MSE $=$ Mean Square Error; and PRESS=Prediction Sum of Square statistic.

Potential aboveground carbon (AGC) sequestered within various agroforestry practices

Most AGC were sequestered by species of trees or shrubs from homegardens, boundary planting; and scattered trees onfarms. On the other hand, woodlots, parklands and alley cropping systems accounted for less than 100 tons/ha quantity of AGC (Table 10).

\begin{tabular}{|c|c|c|c|c|c|}
\hline Practices & N & Min $^{\mathbf{m}}$ & Max $^{\mathbf{m}}$ & Mean \pm Std. Dev (tons/ha) & SE Mean \\
\hline Alley cropping & 15 & 07.5 & 0190.8 & $071.2 \pm 066.1$ & 17.1 \\
\hline Boundary planting & 59 & 11.7 & 1384.7 & $183.1 \pm 282.3$ & 36.8 \\
\hline Home gardens & 135 & 06.4 & 1637.9 & $142.5 \pm 217.1$ & 18.7 \\
\hline Parklands fodder & 41 & 08.5 & 0313.4 & $072.2 \pm 077.9$ & 12.2 \\
\hline Scattered trees & 51 & 06.4 & 2516.8 & $132.2 \pm 426.8$ & 59.8 \\
\hline woodlots & 54 & 10.6 & 0589.5 & $097.6 \pm 134.0$ & 18.2 \\
\hline
\end{tabular}

$\mathrm{N}=$ total number of trees, $\mathrm{Std} . \mathrm{Dev}=$ standard deviation, $\mathrm{SE}=$ standard error, $\mathrm{Min}^{\mathrm{m}}=$ minimum, $\mathrm{Max}^{\mathrm{m}}=$ maximum

Table 10:- Carbon sequestered within Agroforestry Practices in Rajaf County 
Potential aboveground carbon (AGC) sequestered at tree species level

On average; A. seberiana, B. ethiopum, D. quinqueseta, K. africana, T. indica, M. indica, H. artocarpus and B. paradoxum displayed high levels of carbon sequestration due to their characteristic heavy crown cover (Table 11). In contrast, species characterized with low crown cover and scattered distribution such as C. limon, T. grandis, P. guajava, S. siamea, B. aegyptiaca and $A$. indica contributed moderately less to the overall carbon sequestered within the AF farms even though they were inventoried in large numbers.

\begin{tabular}{|c|c|c|c|c|c|c|}
\hline Species Name & Family name & $\mathbf{N}$ & $\operatorname{Min}^{m}$ & $\operatorname{Max}^{\mathrm{m}}$ & $\begin{array}{c}\text { Mean } \pm \text { Std.Dev } \\
\text { (tons/ha) }\end{array}$ & SE Mean \\
\hline Borassus ethiopum & Arecaceae & 8 & 067.9 & 2517.0 & $761.0 \pm 923.0$ & 326.0 \\
\hline Vitellaria paradoxa & Sapotaceae & 5 & 119.0 & 1385.0 & $442.0 \pm 533.0$ & 239.0 \\
\hline Kigelia africana & Bignoniaceae & 7 & 021.3 & 0634.9 & $328.2 \pm 156.2$ & 059.0 \\
\hline Mangifera indica & Anacardiaceae & 73 & 033.4 & 1637.9 & $234.9 \pm 290.5$ & 034.0 \\
\hline Tarmarindus indica & Fabaceae & 7 & 178.0 & 0531.5 & $189.4 \pm 192.2$ & 072.7 \\
\hline Acacia nilotica & Fabaceae & 2 & 016.2 & 0219.1 & $174.4 \pm 063.3$ & 044.8 \\
\hline Acacia seberiana & Fabaceae & 16 & 129.6 & 0658.2 & $158.9 \pm 189.2$ & 047.3 \\
\hline Heterophyllus artocarpus & Moraceae & 7 & 022.4 & 0589.5 & $137.1 \pm 205.5$ & 077.7 \\
\hline Tectona grandis & Verbenaceae & 50 & 011.1 & 0468.0 & $112.8 \pm 129.7$ & 018.3 \\
\hline Balanites aegyptiaca & Zygophyllaceae & 7 & 007.5 & 0288.6 & $088.4 \pm 098.8$ & 037.4 \\
\hline Psidium Guajava & Myrtaceae & 44 & 032.0 & 0199.9 & $063.2 \pm 056.4$ & 008.5 \\
\hline Sclerocarya birrea & Anacardiaceae & 2 & 021.6 & 0066.8 & $060.8 \pm 008.5$ & 006.0 \\
\hline Azadiractha indica & Meliaceae. & 8 & 029.3 & 0103.6 & $051.3 \pm 026.0$ & 009.2 \\
\hline Ziziphus abyssinica & Rhamnaceae & 6 & 019.2 & 0083.7 & $051.3 \pm 023.0$ & 009.4 \\
\hline Persea americana & Lauraceae & 3 & 006.4 & 0084.5 & $049.5 \pm 030.3$ & 017.5 \\
\hline Senna siamea & Fabaceae & 40 & 054.8 & 0150.9 & $035.4 \pm 031.8$ & 005.0 \\
\hline Combretum spp & Combretaceae & 3 & 020.9 & 0049.0 & $031.5 \pm 015.6$ & 008.9 \\
\hline Anona senegalensis & Anonaceae & 2 & 010.6 & 0031.8 & $026.3 \pm 007.7$ & 005.4 \\
\hline Citrus limon & Rutaceae & 53 & 006.4 & 0176.2 & $023.5 \pm 031.6$ & 004.3 \\
\hline Bridelia micrantha & Phyllanthaceae & 2 & 010.9 & 0019.2 & $015.0 \pm 005.9$ & 004.1 \\
\hline Citrus sinensis & Rutaceae & 6 & 006.6 & 0027.5 & $014.9 \pm 008.0$ & 003.3 \\
\hline Dombeya. quinqueseta & Sterculiaceae & 1 & 118.0 & 0118.0 & $118.0 \pm 000.0$ & $*$ \\
\hline Piliostigma reticulata & Fabaceae & 1 & 012.2 & 0055.3 & $055.3 \pm 000.0$ & $*$ \\
\hline Ximenia americana & Olacaceae & 1 & 055.3 & 0020.5 & $020.5 \pm 000.0$ & $*$ \\
\hline Grawiya mollis & Tiliaceae & 1 & 020.5 & 0012.2 & $012.2 \pm 000.0$ & $*$ \\
\hline
\end{tabular}

Table 11:- Carbon sequestered (tons/ha) at species level

* means one or no replicates

$\mathrm{N}=$ total number of trees, Std.Dev=standard deviation, $\mathrm{SE}=$ standard error, $\mathrm{Min}^{\mathrm{m}}=$ minimum, $\mathrm{Max}^{\mathrm{m}}=$ maximum

Aboveground carbon (AGC) sequestered of agroforestry tree species at site-specific level

- AGC sequestered from agroforestry tree species at Gumbo sites

In Gumbo, the AGB show that most of the AF tree species displayed higher contributions to AGC sequestered compared to the other three sites. Gumbo was characterized with more native and exotic AF tree species that were either retained or planted. The highest quantity of more than 2000 tons/ha of AGC was recorded only for B. ethiopum. Five other species, T. indica, B. aegyptica, K. africana, A. seberiana and T. grandis, made relatively moderate contributions to AGC (Figure 1). 


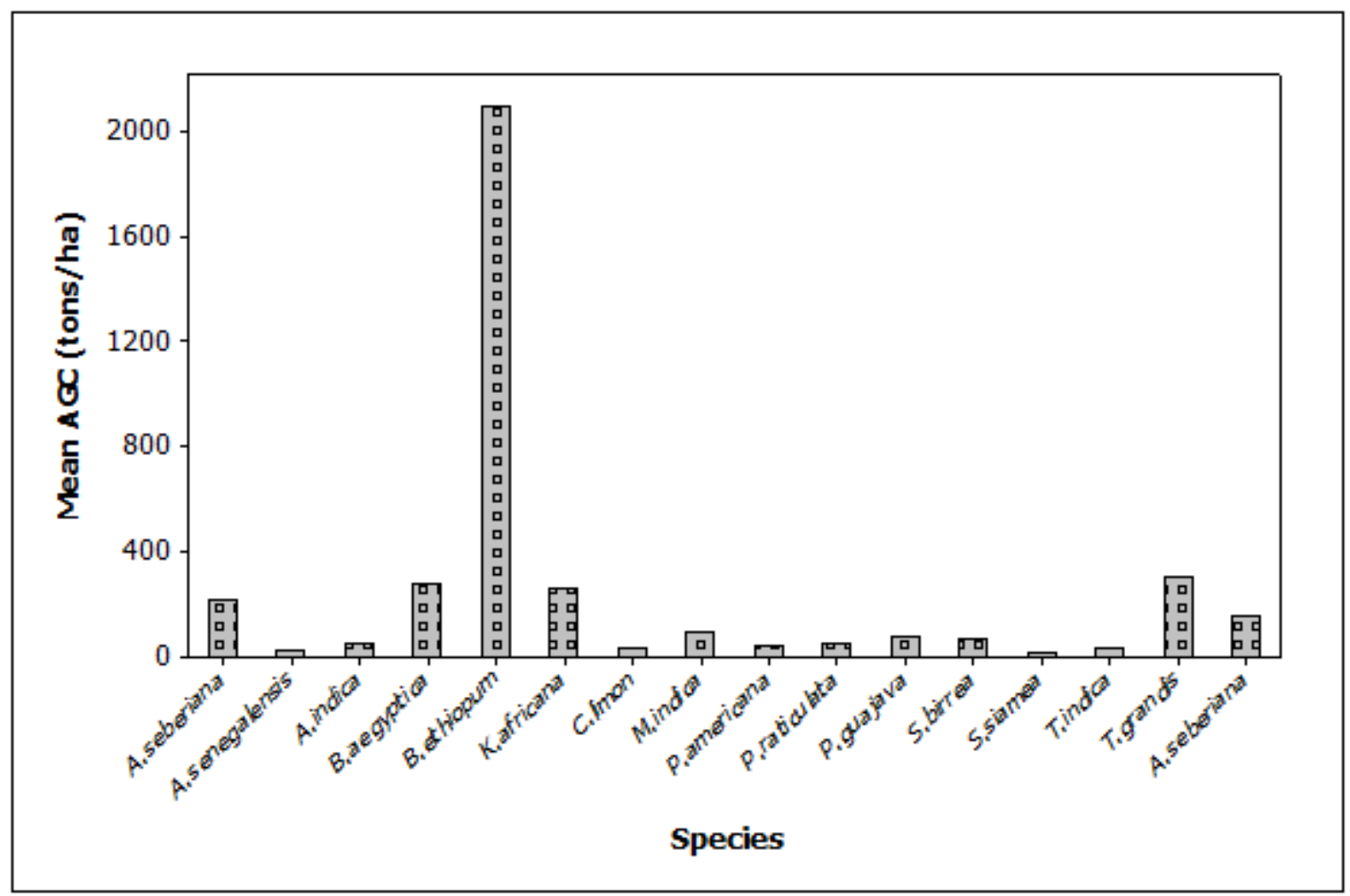

Fig 1:- AGC sequestered by AF tree species at Gumbo site

- AGC sequestered from agroforestry tree species at Kolye East sites

The trend in contributions of AGB to AGC sequestered in Kolye East site was similar to that observed for Gumbo although the AGC sequestered in Kolye East was relatively lower. Kolye East was characterized with more dominant AF tree species that mostly comprised of the natural tree and shrubs species including V.paradoxa, B. ethiopum, K. africana, D. quinqueseta, S. birrea, and Z. abyssinica (Figure 2).

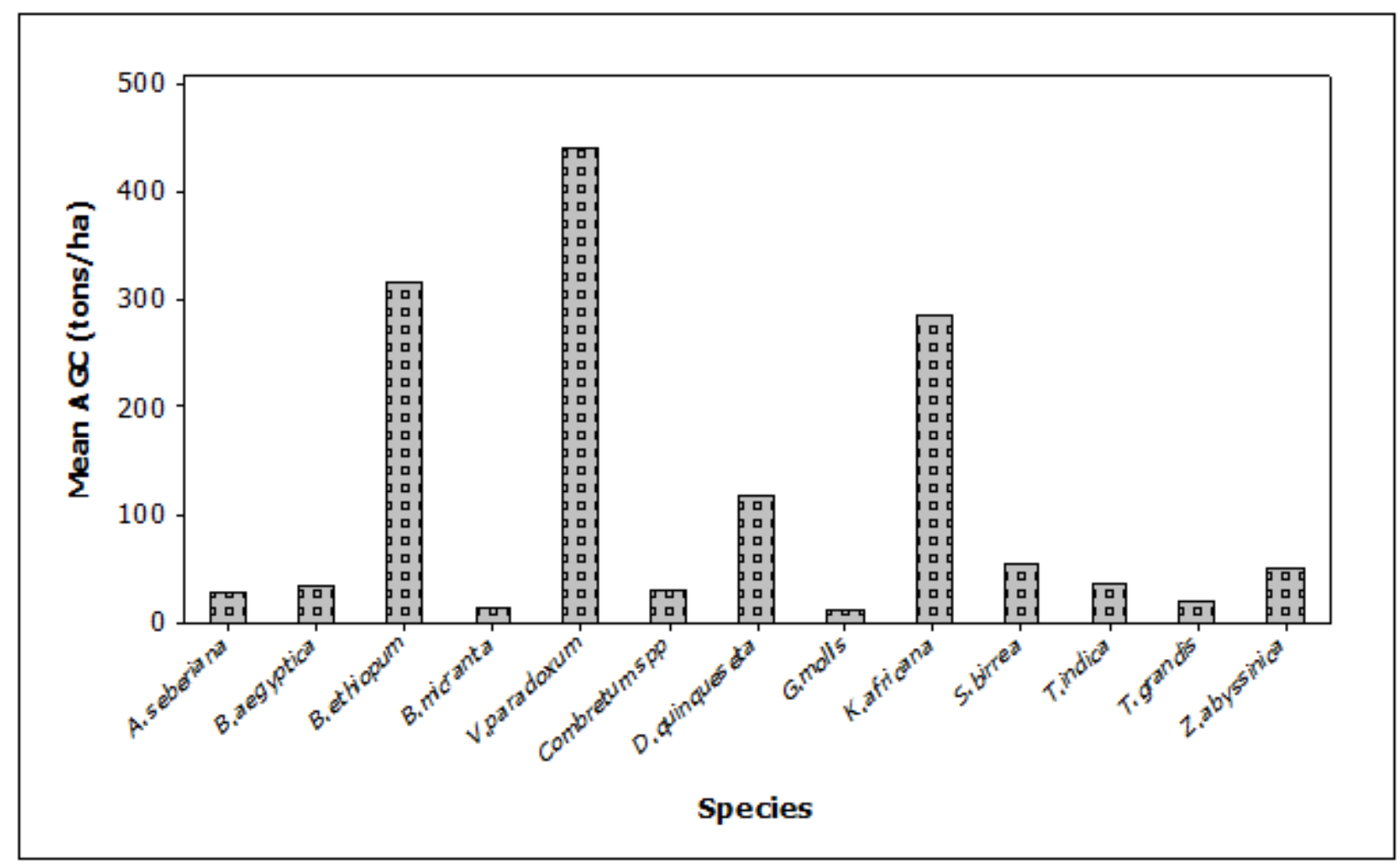

Fig 2:- AGC sequestered by AF tree species at Kolye East 
- AGC sequestered from agroforestry tree species at Kolye West sites

Kolye West site had very few but highly dominant planted AF tree species. The graph shows a low contribution of AGB of the tree species to AGC compared to Gumbo and Kolye East sites. The tree species site has $k$. africana, was the main dominant tree species although most of the tree species were planted. In contrast to the other sites, fewer natural AF trees, including $K$. africana, $M$. indica and S. siamea with relatively moderate contributions of AGB to AGC (ranging from 100 to 400 tons/ha) were recordedin Kolye West. AF tree species with AGB to AGC sequestered-contributions of less than 100 tons/ha included T. grandis, $P$. guajava and A. indica (Figure 3).

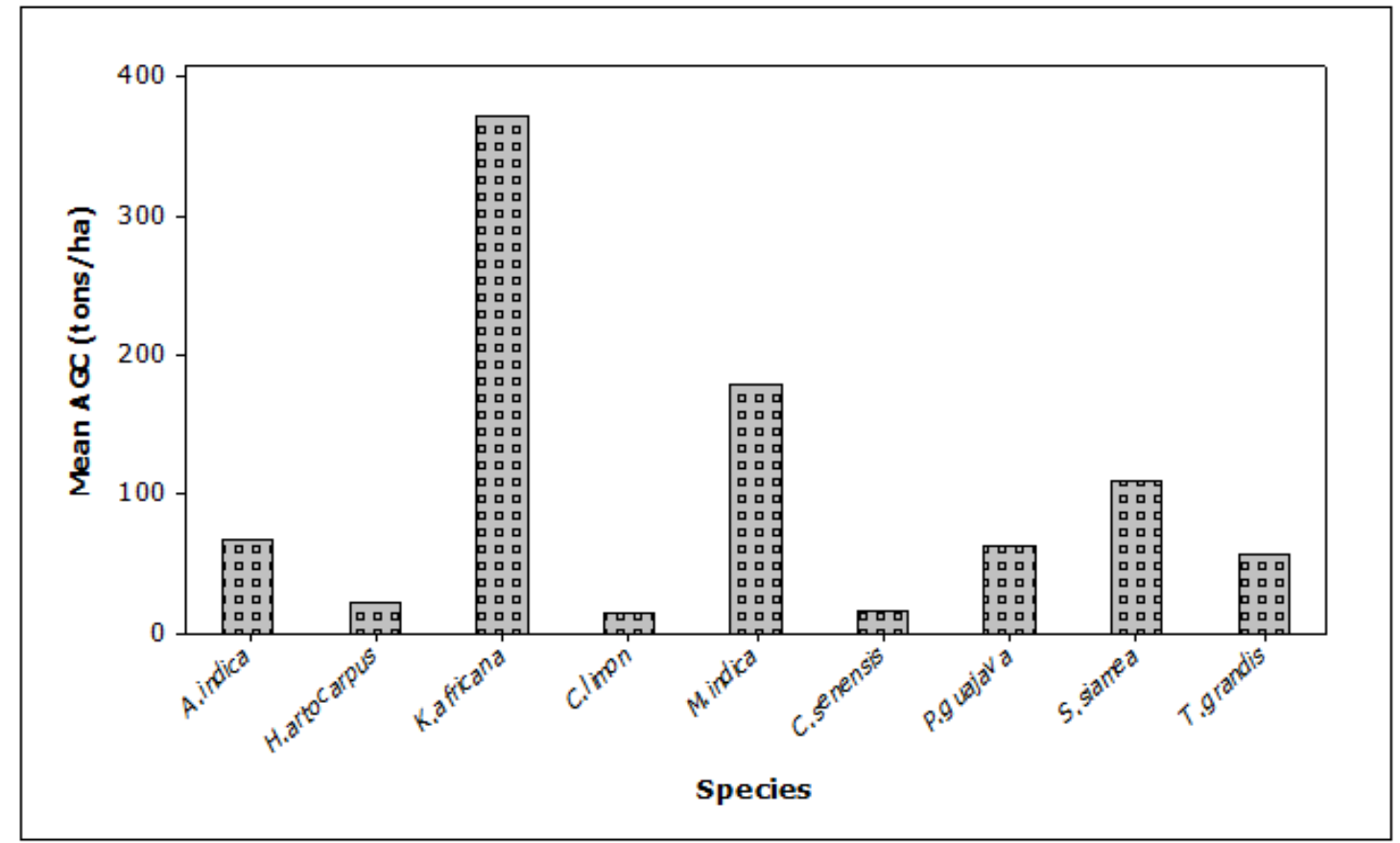

Fig 3:- AGC sequestered by AF tree species at Kolye West

- AGC sequestered from agroforestry tree species at Tokiman (Island) sites

Tokiman islands site was characterized with a rear agro-ecology with $M$. indica and A. nilotica and A. seberiana being the only dominant tree species. The AF tree species with $H$. artocarpus (Jack fruit) and $P$. guajava, although not dominant in the site, were planted along the shores of the Nile river. Most of the few AF tree and shrubs species were comprised of planted fruit trees. The trend of AGB contribution to AGC sequestered in Tokiman was similar to that observed in Kolye West (Figure 4).

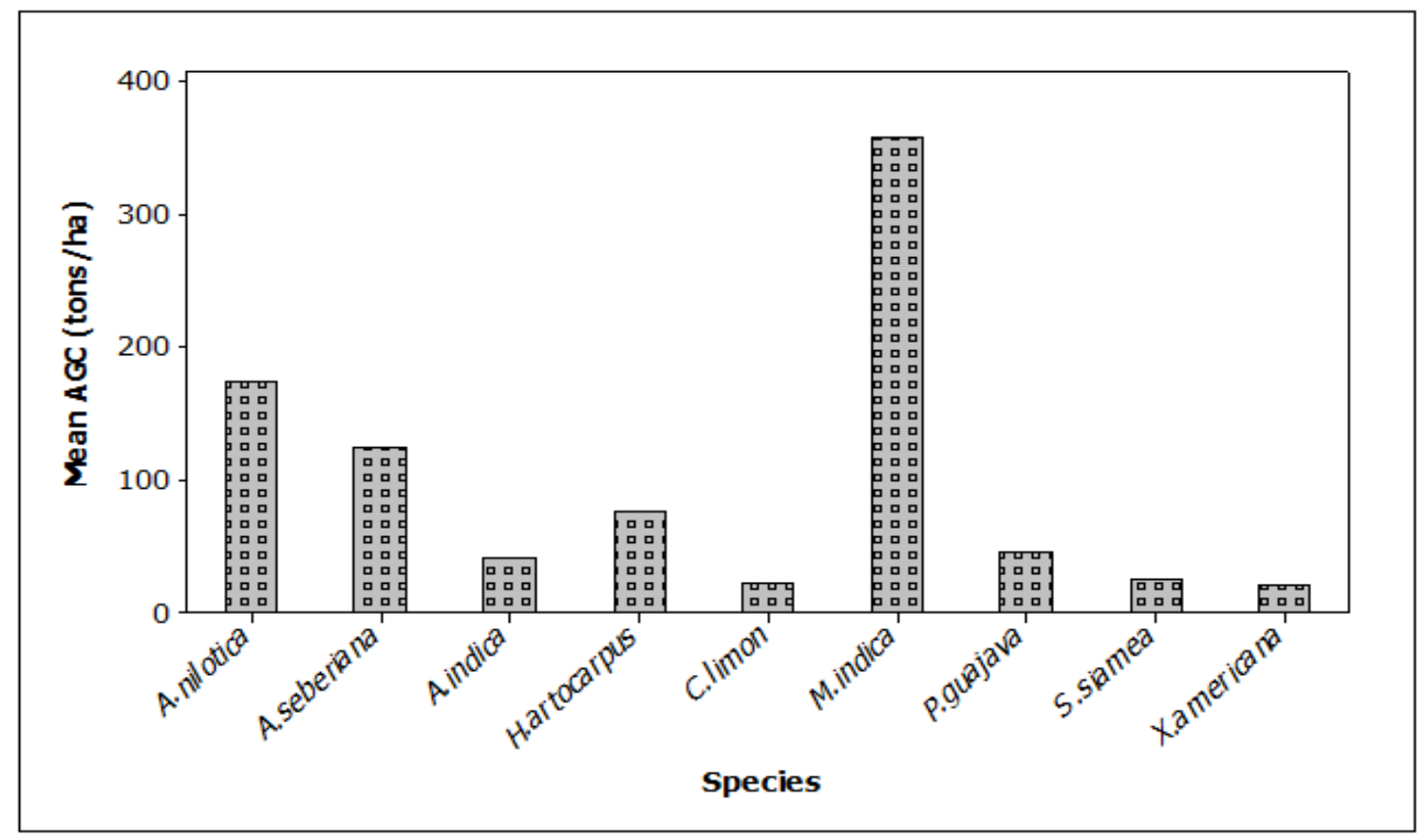

Fig 4:- AGC sequestered by AF tree species in Tokiman 


\section{DISCUSSION}

\section{$>$ The diversity, richness and abundance of on-farm tree species}

The species richness revealed only 25 main species in Gumbo, Tokiman, Kolye East and Kolye West sites, which were more visible and abundant in the AF practices of home-gardenings, boundary planting and scattered trees farming. Shannon diversity index was calculated with its assumption that all species are represented in a sample and are randomly sampled [36]. This could easily be compared by its relationship with dominance index because it gives more weight to common or dominant species. In this case, a few rare species with only a few representatives will not affect the diversity [42].

The average values of Shannon-Weiner (H') indices for Gumbo and Tokiman were relatively lower compared to that for Kolye East and Kolye West, an indication of less diverse communities with few AF tree species in Gumbo and Tokiman sites. While Shannon-Weiner indices for Kolye East and Kolye West were relatively higher than that for Gumbo and Tokiman sites, an indication of moderate levels of diversity and evenness of AF trees. Values of Shannon-Weiner index ranging from 3 or more indicates higher level of diversity and even distribution of tree species [43]. The overall average of Shannon-weiner diversity index (H') and evenness of the woody species were 1.976 and 0.809 , respectively. Nevertheless, this overall calculated average diversity indices falls within the normal range of Shannon-Weiner diversity index, as the value lie between 1.5 and 3.5 and rarely exceed 4.5. The average evenness value of 0.809 in this study was similar to those reported from other agroforestry sites [44]. Thus, the average value of Shannon-weiner diversity index for the four sites showed a moderate diversity of the tree species available $(\mathrm{H}=1.976)$. This on the other hand reflects on average dominance of such tree species. Although Simpson index was not calculated, its relationship with Shannon diversity index is feasible. According to reference [45], if low diversity means high dominance, and high diversity is an indication of low dominance of AF tree species, then moderate diversity should indicate moderate dominance. Therefore, since the average total computed value of $\mathrm{H}$ (1.976) is said to be moderately diverse, then its dominance within the communities is comparable or relative to one another. This indicates a relative variability in distribution of individuals among various species of trees on-farms. The reasons for the variability in diversity of these AF tree species could be attributed to ecological variability which influences their distribution. Most AF tree species on-farms were least diverse and abundant. The reasons could be due to lack of ecological adaptability and anthropogenic activities such as clearing land for agriculture, settlements, overexploitation, deforestations and over grazing of animals that destroy tree crops on farms. Local climatic conditions and variations with high rate of anthropogenic disturbances such as human encroachment and livestock grazing that are common in the study area were among the major factors responsible for variations in species diversity and evenness in the given farms[43]. While high diversity and abundance of other AF species such as M.indica, C.limon, P.guajava and T.grandis could be due to site suitability and adaptability; and moreover, their indigenous interest to plant or retain them for future production of fruits for food and timber for construction could be one of the reasons.

\section{$>$ Aboveground biomass and their estimating models for appropriate selection}

The average DBH and height of AF trees calculated were very low although their average crown width was high, an indication that the trees inventoried were young, few and sparsely distributed within the AF farms. The reduced number and retarded growth of the tree species could be due to poor climatic conditions and human activities such as overgrazing, deforestations, bush burning and land degradation practices. Despite having short, large and differing crown widths, that depicted the existence of large trees in the AF farms, various existing allometric models were used for comparison of outcomes for selection of the best models [46].

By comparing AGB estimating models when tree total height is available, allometric equations of Chave et al.(2014) and Brown(1989) were selected as the best models for estimation of AGB which was subsequently used for calculating AGC sequestered. The inclusion of total tree height and tree trunk diameter in allometric models usually yields less biased estimates as compared to pantropical AGB allometries although it is difficult to measure accurately especially in closed canopies of mixed forests [22]. Results from models of Chave et al.(2005); Ketterings et al.(2001) and Ponce (2004) had high mean variations, which could possibly be due to the diameter tapering coefficients of these three models and also the fact that tree height is not included in these models [47]. However, in this study, tree height measurement was included to form basis of mixed forest equations.

\section{> Potential carbon sequestered from various agroforestry farms}

At systems-specific level, most carbon sequestered was accrued from boundary planting, homegardens spp, and scattered trees in farms with little carbon stocks generated by woodlots, parklands and alley planting. At site-specific level, unlike in other AF farm sites, the results depicted that Gumbo site had more available tree species. This had yielded to more AGB estimates that contributed much equally to AGC sequestered due to their inclusiveness [48]. Both Gumbo and Kolye East sites had almost shown similar trend of amount of AGC sequestered with more dominant AF tree species available [49].

Despite having very few but highly dominant AF tree species, Kolye west site had also shown a moderate contribution of AGB to AGC. This could be due to differences in site suitability and ecological adaptability of those tree species [49]. The AGB contribution to AGC sequestered were significantly and considerably low for Tokiman as compared to other three AF sites [50].This could be due to natural disasters such as massive flooding 
along the shores of River Nile and serious anthropogenic practices that causes soil degradation and washes vegetation away [51]. This finding is also in line with [52], who argued that forest land and shrub bush land cover have decreased while cultivated land and settlements have increased within the recent years signifying the rate of decline of natural vegetation in the catchment areas as population and settlements are rising. This could be attributed to population pressure, drought, flood, overgrazing, etc. Therefore, overall agroforestry carbon stocks for the whole study sites of Rajaf County were low. This low AGC signified a higher level of interference and biomass extraction by the local communities.

\section{CONCLUSIONS}

In many agroforestry systems, trees are highly valued for their great potential to mitigate emissions of atmospheric gases. This is important as they conserve carbon reservoirs and improve carbon storage in vegetations and land cover for climate stability for different land uses. The above ground biomass is the major part of tree variables for initially estimating their potential for carbon stocks and sequestration in most mixed ecosystems. In most agroforestry farms, young and maturing trees (Dbh $\geq 5 \mathrm{~cm}$ ) represent more than $70 \%$ of total above ground biomass (TAGB). Apparently, trees inventoried were young and maturing with mean $\mathrm{DBH}, \mathrm{H}$ and $\mathrm{CR}$ of $13 \mathrm{~cm}$, $6 \mathrm{~m}$ and $3.3 \mathrm{~m}$ respectively. These AF tree species were found to be moderately diverse although diversity is varied from site to site, species to species and how each species adapted to a specific ecological location compared to other communities. The species at the sites do not poses complete evenness but appeared to be moderately distributed.

Based on the model selection criteria, models from Chave et al., (2014) and Brown, (1989) were used for estimating the AGB of AF tree species because they had relatively higher coefficient of multiple determination $\left(R^{2}\right)$, lower mean square error (MSE) and predicted sums of square (PRESS). Based on the findings in this study, we also concluded that most carbon sequestration stock was accrued from the AF practices boundary planting, homegardenings, and scattered trees on-farms. The AF carbon stocks and productivity for Kolye west and Tokiman were significantly lower compared to Gumbo and Kolye East sites.

The study revealed that levels of stabilization of atmospheric carbon dioxide are lower in South Sudan although the study is first of the kind. There is being global efforts by most developmental organizations operating within the region, however, most of these efforts on climate change are not feasible. In Rajaf County, over 50\% carbon sequestered is lost due to massive anthropogenic activities this has left South Sudan in general at a risk of land degradation, and threat to extinction of most natural resources including lost of agricultural productivity.

\section{Conflict of Interest}

The authors declared, there were no conflicts of interest found from the study.

\section{ACKNOWLEDGEMENTS}

The authors would like to acknowledge the financial support rendered to them by NORHED through SUDD Project of the University of Juba for the field work coverage. Nevertheless, we are also very thankful to the Ministry of Forestry and Environment of Republic of South Sudan and the county administration of Rajaf for granting us permission to access various household agroforestry farms. Finally, we thanked the local people, household representatives and the community leaders of Rajaf County for their enormous backup and willingness to take part in the study.

\section{REFERENCES}

[1]. Pachauri RK, Allen MR, Barros VR, Broome J, Cramer W, Christ R, Church JA, Clarke L, Dahe Q, Dasgupta P, Dubash NK. Climate change 2014: synthesis report. Contribution of Working Groups I, II and III to the fifth assessment report of the Intergovernmental Panel on Climate Change. Ipcc; 2014.

[2]. Ebi KL, Hallegatte S, Kram T, Arnell NW, Carter TR, Edmonds J, Kriegler E, Mathur R, O’Neill BC, Riahi K, Winkler H. A new scenario framework for climate changeresearch: background, process, and future directions. Climatic Change. 2014 Feb 1;122(3):363-72.

[3]. Jat ML, Dagar JC, Sapkota TB, Govaerts B, Ridaura SL, Saharawat YS, Sharma RK, Tetarwal JP, Jat RK, Hobbs H, Stirling C. Climate change and agriculture: adaptation strategies and mitigation opportunities for food security in South Asia and Latin America. InAdvances in agronomy 2016 Jan 1 (Vol. 137, pp. 127-235). Academic Press.

[4]. FAO: Trees Outside Forests. Conservation Guide No. 35. Forest Conservation Service, 2001.

[5]. Ekoungoulou R, Niu S, Loumeto JJ, Ifo SA, Bocko YE, Mikieleko FE, Guiekisse ED, Senou H, Liu $\mathrm{X}$. Evaluating the carbon stock in above-and belowground biomass in a moist central African forest. Applied Ecology and Environmental Sciences. 2015;3(2):51-9.

[6]. Pan Y, Birdsey RA, Fang J, Houghton R, Kauppi PE, Kurz WA, Phillips OL, Shvidenko A, Lewis SL, Canadell JG, Ciais P. A large and persistent carbon sink in the world's forests. Science. 2011 Aug 19;333(6045):988-93.

[7]. IPCC (Intergovernmental Panel on Climate Change). Climate change 2001: the scientific basis. Contribution of working group I to the third assessment report of the intergovernmental panel on climate change, 881. 2001. 
[8]. Baccini A, Laporte N, Goetz SJ, Sun M, Dong H. A first map of tropical Africa's above- ground biomass derived from satellite imagery. Environmental Research Letters. 2008 Dec 10;3(4):045011.

[9]. Houghton RA, Hall F, Goetz SJ. Importance of biomass in the global carbon cycle. Journal of Geophysical Research: Biogeosciences. 2009 Jun $1 ; 114(\mathrm{G} 2)$.

[10]. Fousseni F, Xiuhai Z, Chunyu Z, Kperkouma W, Koffi A. Ecological and numerical analyses of plant communities of the most conserved protected area in North-Togo. International Journal of Biodiversity and Conservation. 2010 Nov;2(11):359-69.

[11]. Maina EW, Odera PA, Kinyanjui MJ. Estimation of above ground biomass in forests using Alos Palsar data in Kericho and Aberdare ranges.

[12]. Lu D, Batistella M, Moran E. Satellite estimation of aboveground biomass and impacts of forest stand structure. Photogrammetric Engineering \& Remote Sensing. 2005 Aug 1;71(8):967-74.

[13]. Buyinza J, Tumwebaze S, Namaalwa J, Byakagaba P. Above-ground biomass and carbon stocks of different land cover types in Mt. Elgon, Eastern Uganda.

[14]. Nakakaawa CA, Vedeld PO, Aune JB. Spatial and temporal land use and carbon stock changes in Uganda: implications for a future REDD strategy. Mitigation and Adaptation Strategies for Global Change. 2011 Jan 1;16(1):25-62.

[15]. Agevi H, Onwonga R, Kuyah S, Tsingalia M. Carbon stocks and stock changes in agroforestry practices: a review.

[16]. Negash M, Kanninen M. Modeling biomass and soil carbon sequestration of indigenous agroforestry systems using CO2FIX approach. Agriculture, Ecosystems \& Environment. 2015 May 1;203:14755.

[17]. Ramachandran Nair PK, Mohan Kumar B, Nair VD. Agroforestry as a strategy for carbon sequestration. Journal of plant nutrition and soil science. 2009 Feb;172(1):10- 23.

[18]. Mbow C, Smith P, Skole D, Duguma L, Bustamante M. Achieving mitigation and adaptation to climate change through sustainable agroforestry practices in Africa. Current Opinion in Environmental Sustainability. 2014 Feb 1;6:8-14.

[19]. Mbow C, Van Noordwijk M, Luedeling E, Neufeldt H, Minang PA, Kowero G. Agroforestry solutions to address food security and climate change challenges in Africa. Current Opinion in Environmental Sustainability. 2014 Feb 1;6:61-7.

[20]. Chave J, Réjou-Méchain M, Búrquez A, Chidumayo E, Colgan MS, Delitti WB, Duque A, Eid T, Fearnside PM, Goodman RC, Henry M. Improved allometric models to estimate the aboveground biomass of tropical trees. Global change biology. $2014 \quad$ Oct;20(10):3177-90.

[21]. Zhang C, Peng DL, Huang GS, Zeng WS. Developing aboveground biomass equations both compatible with tree volume equations and additive systems for single-trees in poplar plantations in Jiangsu Province, China. Forests. 2016 Feb;7(2):32.

[22]. Hunter MO, Keller M, Vitoria D, Morton DC. Tree height and tropical forest biomass estimation. Biogeosciences. 10: 8385-8399. 2013.

[23]. Baccini AG, Goetz SJ, Walker WS, Laporte NT, Sun M, Sulla-Menashe D, Hackler J, Beck PS, Dubayah R, Friedl MA, Samanta S. Estimated carbon dioxide emissions from tropical deforestation improved by carbon-density maps. Nature climate change. 2012 Mar;2(3):182-5.

[24]. Kuyah S, Rosenstock TS. Optimal measurement strategies for aboveground tree biomass in agricultural landscapes. Agroforestry systems. 2015 Feb 1;89(1):125- 33.

[25]. Agevi H, Wabusya M, Kigen C. Diversity and Biomass Variation in Masinde Muliro University of Science and Technology.

[26]. Basuki TM, Van Laake PE, Skidmore AK, Hussin YA. Allometric equations for estimating the aboveground biomass in tropical lowland Dipterocarp forests. Forest ecology and management. 2009 Mar 31;257(8):1684-94.

[27]. Kuyah S, Dietz J, Muthuri C, Jamnadass R, Mwangi $\mathrm{P}$, Coe R, Neufeldt H. Allometric equations for estimating biomass in agricultural landscapes: II.

Belowground biomass. Agriculture, ecosystems \& environment. 2012 Sep 1; 158:225-34.

[28]. Sudan Population \& Housing Census, 2008.

[29]. Mbwiga J. Classification of chagga agroforestry homegardens and their contributions to food, income and wood energy to communities of Rombo District, Tanzania (Doctoral dissertation, Sokoine University of Agriculture).

[30]. Shilabu MD. The contribution of agroforestry to household food security and income generation in Maswa District, Shinyanga region (Doctoral dissertation, Sokoine University of Agriculture (SUA)).

[31]. Donat MG, Peterson TC, Brunet M, King AD, Almazroui M, Kolli RK, Boucherf D, Al-Mulla AY, Nour AY, Aly AA, Nada TA. Changes in extreme temperature and precipitation in the Arab region: long-term trends and variability related to ENSO and NAO. International Journal of Climatology. 2014 Mar 15;34(3):581-92.

[32]. Heiskanen J, Pellikka P, Betemariam EA, Packalen P. Field measurement guidelines for aboveground biomass and fuel wood stocks.

[33]. Ekoungoulou R, Liu X, Ifo SA, Loumeto JJ, Folega F. Carbon stock estimation in secondary forest and gallery forest of Congo using allometric equations. International Journal of Scientific and Technology Research. 2014;3(3):465-74.

[34]. Fousseni F, Xiuhai Z, Chunyu Z, Kperkouma W, Koffi A. Ecological and numerical analyses of plant communities of the most conserved protected area in North- Togo. International Journal of Biodiversity and Conservation. 2010 Nov;2(11):35969. 
[35]. Feldpausch TR, Lloyd J, Lewis SL, Brienen RJ, Gloor M, Monteagudo Mendoza A, LopezGonzalez G, Banin L, Abu Salim K, Affum-Baffoe K, Alexiades M. Tree height integrated into pantropical forest biomass estimates. Biogeosciences. 2012 Aug 27:3381- 403.

[36]. Molla A, Kewessa G. Woody species diversity in traditional agroforestry practices of Dellomenna District, Southeastern Ethiopia: Implication for maintaining native woody species. International Journal of Biodiversity. 2015;2015.

[37]. Chave J, Andalo C, Brown S, Cairns MA, Chambers JQ, Eamus D, Fölster H, Fromard F, Higuchi N, Kira T, Lescure JP. Tree allometry and improved estimation of carbon stocks and balance in tropical forests. Oecologia. 2005 Aug 1; 145(1):87-99.

[38]. Ketterings QM, Coe R, van Noordwijk M, Palm CA. Reducing uncertainty in the use of allometric biomass equations for predicting above-ground tree biomass in mixed secondary forests. Forest Ecology and management. 2001 Jun 1;146(1-3):199209.

[39]. Brown S, Gillespie AJ, Lugo AE. Biomass estimation methods for tropical forests with applications to forest inventory data. Forest science. 1989 Dec 1;35(4):881-902.

[40]. Ponce H. Assessing carbon stocks and modelling winwin scenarios of carbon sequestration through land-use changes. Assessing carbon stocks and modelling win- win scenarios of carbon sequestration through land-use changes.. 2004.

[41]. Djomo AN, Ibrahima A, Saborowski J, Gravenhorst G. Allometric equations for biomass estimations in Cameroon and pan moist tropical equations including biomass data from Africa. Forest Ecology and Management. 2010 Oct 15; 260(10):1873-85.

[42]. Uthappa AR, Chavan SB, Singh M, Sridhar KB, Dev I, Ram A, Sathish BN, Kumar M, Dwivedi RP, Singh R, Singh RK. Tree diversity in ravines and their rehabilitation through agroforestry interventions in Bundelkhand Region of India. Indian Journal of Agroforestry. 2016;18(1):77-83.

[43]. Woldearegay M, Woldu Z, Lulekal E. Species diversity, population structure and regeneration status of woody plants in Yegof dry afromontane forest, Northeastern Ethiopia. European Journal of Advanced Research in Biological and Life Sciences. 2018;6(4).

[44]. Zegeye H, Teketay D, Kelbessa E. Diversity and regeneration status of woody species in Tara Gedam and Abebaye forests, northwestern Ethiopia. Journal of Forestry Research. 2011 Sep 1;22(3):315.

[45]. Jew EK, Dougill AJ, Sallu SM, O'Connell J, Benton TG. Miombo woodland under threat: Consequences for tree diversity and carbon storage. Forest Ecology and Management. 2016 Feb 1;361:144-53.

[46]. Goodman RC, Phillips OL, Baker TR. The importance of crown dimensions to improve tropical tree biomass estimates. Ecological Applications. 2014 Jun; 24(4):680-98.
[47]. Huang Y, Lechowicz MJ, Zhou D, Price CA. Evaluating general allometric models: interspecific and intraspecific data tell different stories due to interspecific variation in stem tissue density and leaf size. Oecologia. 2016 Mar 1;180 (3):671-84.

[48]. Yismaw MT, Tadesse B. The Contribution of Agro forestry System to Tree Biodiversity Conservation and Rural Livelihood: The Case of Lay Armachiho District, Gondar, Ethiopia. International Journal of Scientific Research and Management. 2018 May $4 ; 6(05)$.

[49]. Sisay, K., Thurnher, C., Belay, B., Belete, W., Teklehaymanot, T., Habte, K., $\quad$...\&Hasenauer, H. Estimation of aboveground volume, carbon stocks and NPP using terrestrial and satellite data of Amhara region, Ethiopia.In Conference on International Research on Food Security. 2016, September.

[50]. Neumann M, Zhao M, Kindermann G, Hasenauer H. Comparing MODIS net primary production estimates with terrestrial national forest inventory data in Austria. Remote Sensing. 2015 Apr;7(4):3878906.

[51]. Negash M, Starr M. Biomass and soil carbon stocks of indigenous agroforestry systems on the southeastern Rift Valley escarpment, Ethiopia. Plant and Soil. 2015 Aug 1; 393(1- 2):95-107.

[52]. Tesfaye S, Guyassa E, Joseph Raj A, Birhane E, Wondim GT. Land use and land cover change, and woody vegetation diversity in human driven landscape of Gilgel Tekeze Catchment, Northern Ethiopia. International Journal of Forestry Research. 2014; 2014. 